\title{
Triploidy that escaped diagnosis using chromosomal microarray testing in early pregnancy loss: Two cases and a literature review
}

\author{
Ji Eun Park ${ }^{1 \oplus}$, ji Kwon Park ${ }^{1 *} \oplus$, Min Young Kang ${ }^{1 \oplus}$, Hyen Chul jo ${ }^{1 \oplus}$, In $\mathrm{Ae} \mathrm{Cho}^{2 \oplus}$, and Jong Chul Baek ${ }^{1 \oplus}$ \\ ${ }^{1}$ Department of Obstetrics and Gynecology, Gyeongsang National University Changwon Hospital, College of Medicine, Gyeongsang National \\ University, Changwon, Korea \\ ${ }^{2}$ Department of Obstetrics and Gynecology, Gyeongsang National University Hospital, College of Medicine, Gyeongsang National University, \\ jinju, Korea
}

\begin{abstract}
About $15 \%$ to $20 \%$ of all clinically recognized pregnancies result in spontaneous abortion or miscarriage, and chromosomal anomalies can be identified in up to $50 \%$ of first trimester miscarriages. Chromosomal microarray analysis (CMA) is currently considered first-tier testing for detecting fetal chromosomal abnormalities and is supported by the absence of cell culture failure or erroneous results due to cell contamination in pregnancy loss. Triploidy is a lethal chromosome number abnormality characterized by an extra haploid set of chromosomes. Triploidy is one of the most common chromosomal aberrations in first trimester spontaneous abortions. Here, we report two cases of triploidy abortion that were not detected using array comparative genomic hybridization-based CMA. The aim of this report was to remind clinicians of the limitations of chromosomal testing and the misdiagnosis that can result from biased test selection.
\end{abstract}

Key words: Comparative genomic hybridization, Microarray analysis, Karyotyping, Abortion, spontaneous, Triploidy.

\section{Introduction}

An estimated $15 \%$ to $20 \%$ of clinically recognized pregnancies end in miscarriage (loss of pregnancy less than 20 weeks gestation) [1]. The single most common cause of early pregnancy loss is the presence of a fetal chromosomal abnormality, which is responsible for more than $50 \%$ of losses in the first trimester [2]. The diagnosis of chromosomal abnormalities in pregnancy loss is essential to establish the etiology of fetal losses and to assess patients with risks of recurrence. Such a diagnosis can also relieve feelings of guilt associated with pregnancy loss and provide options for prenatal or preimplantation genetic testing in future pregnancies [3].

Triploidy is a chromosomal aberration caused by the presence of an extra haploid chromosome set. Triploidy is the second most frequent chromosomal abnormality in early pregnancy loss with chromosomal abnormalities, following trisomy, and is responsible for about 13\% of these losses [3].

Recent studies have provided support for the utility of chromosomal microarray analysis (CMA) testing in pregnancy loss $[4,5]$. The main reasons are the elimination of cell culture failures, the risk of erroneous results due to maternal cell contamination,

Received: 24 September 2019, Revised: 22 November 2019, Accepted: 22 November 2019, Published: 31 December 2019

*Corresponding author: Ji Kwon Park, M.D., Ph.D. (iD https://orcid.org/0000-0002-9731-190X

Department of Obstetrics and Gynecology, Gyeongsang National University Changwon Hospital, College of Medicine, Gyeongsang National University, 11 Samjeongja-ro, Seongsan-gu, Changwon 51472, Korea.

Tel: +82-55-214-3780, Fax: +82-55-214-3253, E-mail: obgypjk@gnu.ac.kr

Conflict of interest: The authors declare that they do not have any conflicts of interest.

(c) This is an open-access article distributed under the terms of the Creative Commons Attribution Non-Commercial License (http://creativecommons.org/licenses/by-nc/4.0/) which permits unrestricted non-commercial use, distribution, and reproduction in any medium, provided the original work is properly cited.

(c) Copyright 2019 by the Korean Society of Medical Genetics and Genomics 
and the delay of as much as 2 weeks in obtaining conventional karyotyping results. Therefore, the CMA method is increasingly considered the robust choice for the genetic analysis of pregnancy loss. However, CMA also has limitations. Because CMA only identifies the differences reflected by the patient's imbalance compared with the control, a balanced rearrangement is not detected. Specifically, comparative genomic hybridization (CGH)-based arrays cannot detect triploidy. Here, we report two cases of triploidy abortion that were not identifiable using array CGH-based CMA.

\section{Case}

\section{Case 1}

A 37-year-old nulliparous woman was referred for a small gestational sac relative to the expected gestational week $(9+5$ weeks of gestation). She became pregnant by natural ovulation and coitus. Four previous in vitro fertilization-embryo transfers were performed before this natural pregnancy, and all resulted in implantation failure. She denied using teratogenic medications, recent viral infection, diabetes mellitus, or hypertension. She and her husband were non-consanguineous and appeared healthy. There was no family history of congenital malformation. Her husband was 39 years of age. Ultrasonography (USG)

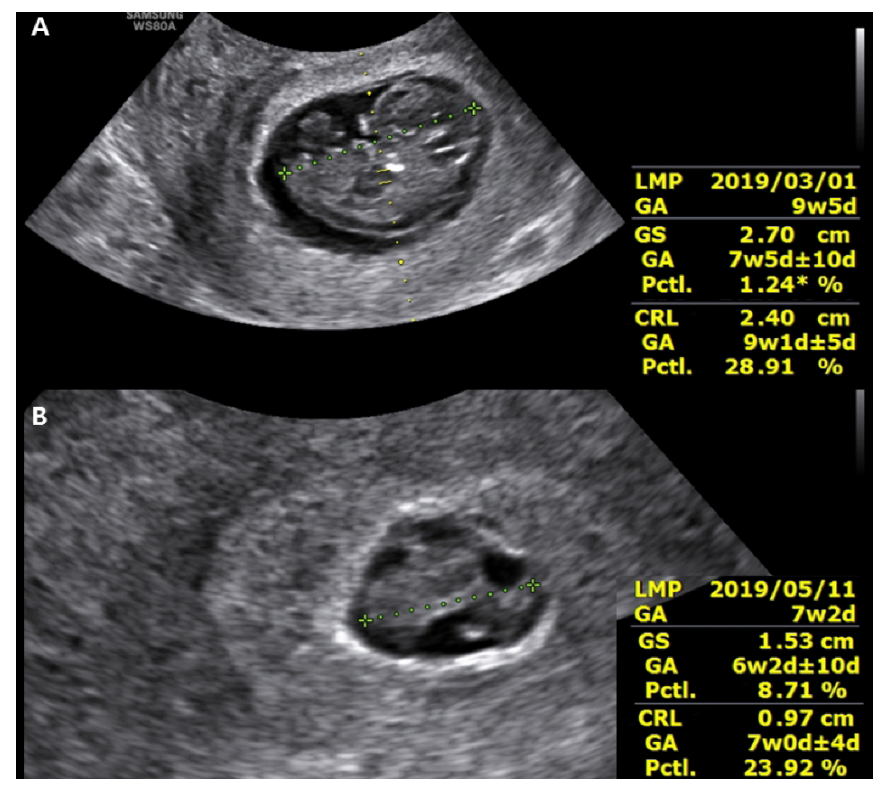

Fig. 1. First ultrasound findings for both cases show small gestational sacs compared with embryo size. (A) In case 1, a 7+5 week sized gestational sac $(27 \mathrm{~mm})$ and $9+1$ week sized embryo $(24 \mathrm{~mm})$ were observed. (B) In case 2, a 6+2 week sized gestational sac $(15.3 \mathrm{~mm})$ and $7+0$ week sized embryo $(9.7 \mathrm{~mm})$ were observed. LMP, last menstrual period; GA, gestational age; GS, gestational sac; Pctl, percentile; CRL, crown rump length. showed a 7+5-week-sized gestational sac (27 mm), 9+1-weeksized embryo (24 mm), but no heartbeat (Fig. 1A). We decided to perform dilatation and evacuation (DEEE) under the diagnosis of early pregnancy loss. In addition, aborted tissue and parental chromosome tests were planned for future pregnancies. D\&E was performed with adjuvant prostaglandin E1 at 10+3 weeks of gestation. Conventional parental karyotyping was performed. The villi of the abortions were genetically tested using two methods: CMA and conventional karyotyping. Both parents had normal chromosome complements in peripheral blood lymphocytes. In the villi of the aborted tissues analyzed by array $\mathrm{CGH}$ (CancerROP, Seoul, Korea), no chromosome alterations were found (Fig. 2A), but conventional karyotyping confirmed triploidy (69, XXX) (Fig. 3A).

\section{Case 2}

A 33-year-old nulliparous woman was referred for a small gestational sac relative to the expected gestational week $(7+2$ weeks of gestation). She became pregnant by natural ovulation and coitus. She denied using teratogenic medications, recent viral infection, diabetes mellitus, or hypertension. She and her husband were non-consanguineous and appeared healthy. There was no family history of congenital malformation. Her husband was 35 years of age. The first USG showed a 5+6-weeksized gestational sac (10.5 mm), 7+1-week-sized embryo (11
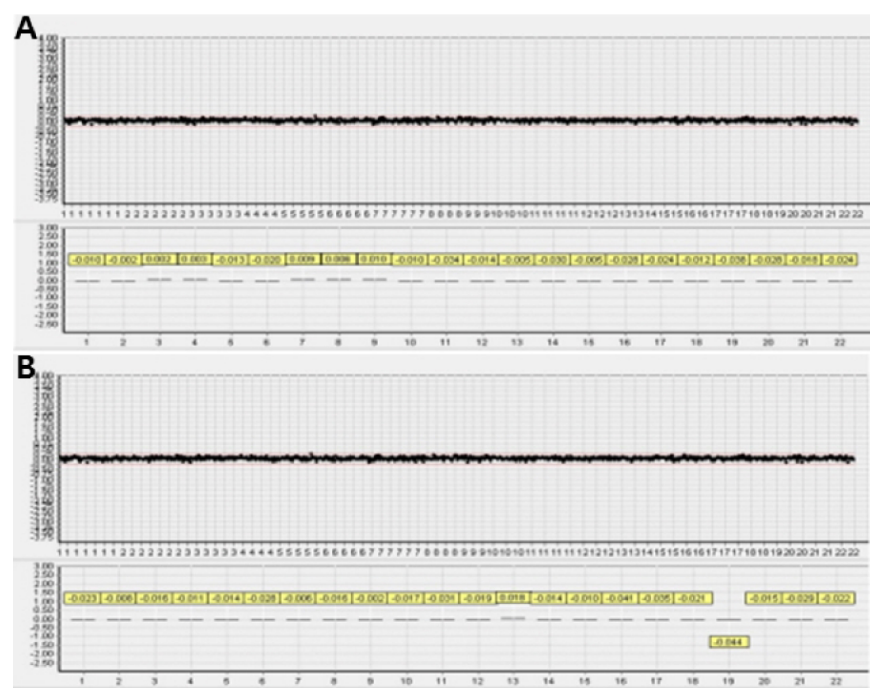

Fig. 2. Comparative genomic hybridization-array-based chromosomal microarray analysis profiles of villus samples collected after spontaneous abortion. The $x$-axis indicates array spots of bacterial artificial chromosome clones ordered from chromosomes 1-22. The y-axis shows the fluorescence ratio of differently labeled sample/control DNA. No losses or gains are observed. (A) Case 1, (B) Case 2. 


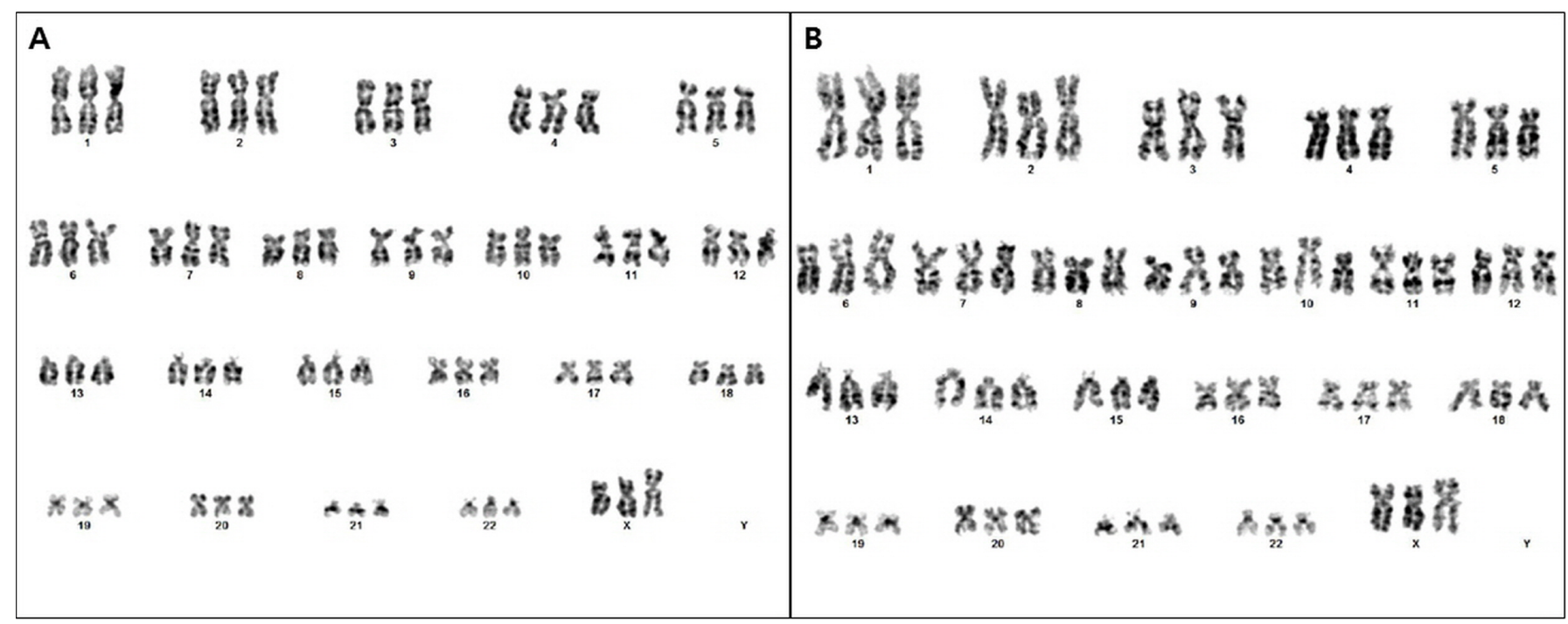

Fig. 3. Conventional cytogenetic direct chorionic villi analysis of the fetuses showed trisomy 69, XXX. (A) Case 1, (B) Case 2.

mm) (Fig. 1B), and a heartbeat was observed. After 10 days, she visited the hospital with vaginal spotting. No fetal heartbeat was observed on the second USG, with a 6+2-week-sized gestational sac (15.3 mm) and 7+0-week-sized embryo (9.7 mm). DEtE was performed under a diagnosis of early pregnancy loss. In addition, aborted tissue chromosome tests were planned for future pregnancies. The DEtE was performed with adjuvant prostaglandin $\mathrm{E} 1$ at $8+5$ weeks of gestation. The aborted tissue was karyotyped using CMA and conventional karyotyping. In the villi of aborted tissue analyzed by array-CGH, no chromosome alterations were found (Fig. 2B), but conventional karyotyping confirmed triploidy (69, XXX) (Fig. 3B).

In both cases, villus samples were collected from the aborted tissue. The samples were obtained by DEtE after spontaneous abortion and collected in sterile containers containing $10 \mathrm{~mL}$ of F-10 (1x) with I-glutamine (Invitrogen, Carlsbad, CA, USA). The villi chromosomes were tested using the following two techniques.

\section{Cytogenetic analysis}

In the genetics laboratory, trained technologists dissected and selected the placental chorionic villi and cultured them in Chang Medium C (Irvine Scientific, Santa Ana, CA, USA) at $37^{\circ} \mathrm{C}$ in a 5\% $\mathrm{CO}_{2}$ incubator. Whenever the material contained chorionic villi, both short- and long-term cultures were set up. All procedures, including cell harvesting, slide preparation, and staining, were conducted following standard protocols. The chromosomes were analyzed after G-banding using Wright stain. At least 20 GTG-banded metaphases were examined and the final assess- ment was made by the cytogenetics laboratory director.

\section{Array CGH}

The array CGH slides (CancerROP) were analyzed using the ChromoFluor image analysis system (Array Analysis; CancerROP). The slides contained 1,440 human bacterial artificial chromosome (BAC) clones including the specific loci of more than 200 chromosomal disorders and 356 cancer-related genes from BAC libraries at an average resolution of 0.1 to $2.3 \mathrm{Mb}$ for the entire genome. Each BAC clone was represented on the array with three spots, and each array was scanned using a GenePix 4000B scanner (Axon Instruments, Foster City, CA, USA) and analyzed with Array Analysis (CancerROP). Normal Korean pooled genomic DNA was used as reference DNA (CancerROP). The green (test) to red (reference) $(G / R$ ) ratios were determined automatically for each sample, and the normalized G/R ratio represented the relative average number of copies of the sequence for those spots that were selected as controls. Spots with $G / R$ ratios of the mean plus 0.25 standard deviations or more (i.e., $G / R \geq 1.25$ ) were considered to show amplification or gain of the indicated copy number, whereas those with $\mathrm{G} / \mathrm{R}$ ratios of the mean minus 0.25 standard deviations or less (i.e., $\mathrm{G} / \mathrm{R} \leq 0.75$ ) were considered to show a loss of copy number.

\section{Discussion}

CMA is a high-resolution technique for measuring the gain and loss of DNA throughout the human genome. CMA provides higher resolution than conventional karyotyping, which enables 
the detection of sub-microscopic deletions and duplications of 50 to $100 \mathrm{~kb}$ [6]. Based on this, the American College of Obstetricians and Gynecologists (ACOG) and Society for Maternal and Fetal Medicine (SMFM) published a statement recommending CMA as the first-line test when prenatal USG shows major fetal abnormalities or fetal demise [7]. This was reinforced by updated ACOG and SMFM practice bulletins, and when fetal structural abnormalities are discovered by USG, CMA should be conducted with the primary test replacing karyotyping [8].

There are two major platforms for CMA: CGH and single nucleotide polymorphism (SNP) arrays. CGH array enables detection of the gain and loss of genomic regions through hybridization of different colored fluorescent-labeled fetal and reference DNA samples. Genomic imbalances can be identified by measuring the signal intensity ratio between the fetal and reference DNA on individual targets and their genomic location after a normalization process. Target DNA fragments aligned on a microarray are called probes, and the resolution of the microarray is determined by the type and number of probes and their distribution on the genome. A typical clinical CGH array contains a few hundred thousand probes, whereas the number of probes on research CGH arrays may reach into the millions [9]. As the intensity of resulting data is typically plotted as a Log2 ratio, where a log2 ratio of zero represents a normal DNA copy number of 2, triploidy cannot be detected by CGH array because the Log2 ratios for a normal diploid sample and a triploid sample are indistinguishable [10]. SNP arrays were originally designed to detect common SNPs and are mainly used for genotyping individuals in genome-wide association studies of multifactorial diseases. However, such platforms can also be used to analyze copy-number variants (CNVs) to compare signal intensities of fetal and reference targets as used in the CGH approach $[11,12]$. SNP arrays can detect triploidy as well as CNVs by assessing the SNP allele patterns on the array, but CGH arrays cannot detect triploidy [9]. In addition, the existing commercial SNP chip is Western-oriented, and has a low genome representation of Asians [13].

Triploidy is one of the most common chromosomal abnormalities; it occurs in $1 \%$ to $2 \%$ of all conceptuses and accounts for more than $10 \%$ of all spontaneous abortions [14]. The primary mechanisms leading to triploidy include dispermy and maternal (digynic triploidy) or paternal (diandric triploidy) meiotic errors [15]. Unlike most chromosomal abnormalities, increased maternal age is not a risk factor for digynic or diandric triploidy. However, there have been several reports of recurrent ( $\geq 3$ times) triploidy [16-18], and one study examined possible genetic vari- ants responsible for this phenotype [19]. These reports support the need for chromosome diagnosis in early pregnancy loss.

In the analysis of early pregnancy loss, the CGH array is limited in that it cannot reliably detect triploidy, which accounts for 10\% of all early pregnancy loss; thus, applying CMA uniformly, without knowledge of each platform, is not a reasonable approach for identifying chromosomal abnormalities in early pregnancy loss. Therefore, before choosing a chromosome test, CMA sub-methods should be given careful consideration, and conventional karyotyping is still important when options are limited. Moreover, because CMA finds genomic imbalances, this technique cannot detect balanced chromosomal rearrangements. In addition, CMA does not provide information about the chromosomal mechanisms of genetic imbalance [20]; for example, if there is a gain of a particular chromosome, CMA cannot distinguish between trisomy and unbalanced Robertsonian translocations, thus there are limitations regarding counseling related to the risk of recurrence. Therefore, excluding conventional karyotyping can have unintended negative consequences.

We report two cases of triploidy that escaped diagnosis using CGH array-based CMA. As mentioned above, because CMA has several platforms and each has advantages and disadvantages, it is important to understand the characteristics and limitations of each platform when selecting a microarray. Thus, in prenatal chromosome testing, particularly in early pregnancy failure, conventional karyotyping is still important and valid in clinical practice. We suggest that providers choose a method based on individual availability and consideration of the strengths and limitations of each test as it applies to each clinical scenario.

\section{References}

1. Steer C, Campbell S, Davies M, Mason B, Collins W. Spontaneous abortion rates after natural and assisted conception. BMJ 1989:299:1317-8.

2. Hassold TJ. A cytogenetic study of repeated spontaneous abortions. Am J Hum Genet 1980;32:723-30.

3. Rosenfeld JA, Tucker ME, Escobar LF, Neill NJ, Torchia BS, McDaniel $L D$, et al. Diagnostic utility of microarray testing in pregnancy loss. Ultrasound Obstet Gynecol 2015;46:478-86.

4. Soler A, Morales $C_{1}$ Mademont-Soler I, Margarit E, Borrell A, Borobio V, et al. Overview of chromosome abnormalities in first trimester miscarriages: a series of 1,011 consecutive chorionic villi sample karyotypes. Cytogenet Genome Res 2017;152:81-9.

5. Sahoo T, Dzidic N, Strecker MN, Commander S, Travis MK, Doherty C, et al. Comprehensive genetic analysis of pregnancy loss by chromo- 
somal microarrays: outcomes, benefits, and challenges. Genet Med 2017;19:83-9.

6. Dugoff L, Norton ME, Kuller JA; Society for Maternal-Fetal Medicine (SMFM). The use of chromosomal microarray for prenatal diagnosis. Am J Obstet Gynecol 2016;215:B2-9.

7. American College of Obstetricians and Gynecologists Committee on Genetics. Committee opinion no. 581: the use of chromosomal microarray analysis in prenatal diagnosis. Obstet Gynecol 2013;122:1374-7.

8. American College of Obstetricians and Gynecologists' Committee on Practice Bulletins-Obstetrics; Committee on Genetics; Society for Maternal-Fetal Medicine. Practice bulletin no. 162: prenatal diagnostic testing for genetic disorders. Obstet Gynecol 2016;127:e108-22.

9. Levy B, Wapner R. Prenatal diagnosis by chromosomal microarray analysis. Fertil Steril 2018;109:201-12.

10. Levy B, Burnside RD. Are all chromosome microarrays the same? What clinicians need to know. Prenat Diagn 2019;39:157-64.

11. McGillivray G, Rosenfeld JA, McKinlay Gardner RJ, Gillam LH. Genetic counselling and ethical issues with chromosome microarray analysis in prenatal testing. Prenat Diagn 2012;32:389-95.

12. Resta N, Memo L. Chromosomal microarray (CMA) analysis in infants with congenital anomalies: when is it really helpful? J Matern Fetal Neonatal Med 2012;25 Suppl 4:124-6.

13. Wong LP, Ong RT, Poh WT, Liu $X_{1}$ Chen $P$, Li R, et al. Deep whole-ge- nome sequencing of 100 southeast Asian Malays. Am J Hum Genet 2013;92:52-66.

14. Hassold T, Chen N, Funkhouser J, Jooss T, Manuel B, Matsuura J, et al. A cytogenetic study of 1000 spontaneous abortions. Ann Hum Genet 1980:44:151-78.

15. Baumer A, Balmer D, Binkert F, Schinzel A. Parental origin and mechanisms of formation of triploidy: a study of 25 cases. Eur J Hum Genet 2000:8:911-7.

16. Brancati F, Mingarelli R, Dallapiccola B. Recurrent triploidy of maternal origin. Eur J Hum Genet 2003;11:972-4.

17. Huang B, Prensky L, Thangavelu M, Main D, Wang S. Three consecutive triploidy pregnancies in a woman: genetic predisposition? Eur J Hum Genet 2004;12:985-6.

18. Pergament $E_{\text {, Confino }}$, Zhang JX, Roscetti L, Xien Chen P, Wellman D. Recurrent triploidy of maternal origin. Prenat Diagn 2000;20:561-3.

19. Filges I, Manokhina I, Peñaherrera MS, McFadden DE, Louie K, Nosova $E_{\text {, et }}$ al. Recurrent triploidy due to a failure to complete materna meiosis II: whole-exome sequencing reveals candidate variants. Mol Hum Reprod 2015;21:339-46.

20. South ST, Lee C, Lamb AN, Higgins AW, Kearney HM; Working Group for the American College of Medical Genetics and Genomics Laboratory Quality Assurance Committee. ACMG Standards and Guidelines for constitutional cytogenomic microarray analysis, including postnatal and prenatal applications: revision 2013. Genet Med 2013;15:901-9 